\title{
Revista Colombiana de

\section{Asociación del tejido adiposo epicárdico con la fibrilación auricular y su pronóstico posterior a la ablación de venas pulmonares}

\author{
Viviana Quintero-Yepes ${ }^{\mathrm{a}, *}$, Mauricio Duque-Ramírez ${ }^{\mathrm{b}}$, Pedro Abad-Díaz ${ }^{\mathrm{c}}$, \\ Sebastián Isaza-Zapata ${ }^{d}$, Laura Duque-González ${ }^{e}$, Elsa M. Vásquez-Trespalacios ${ }^{f}$, \\ Juan C. Díaz-Martínez ${ }^{\mathrm{b}}$, Julián M. Aristizábal ${ }^{\mathrm{b}}$ y William Uribe ${ }^{\mathrm{b}}$
}

a Cardiología clínica, Universidad CES, Medellín, Colombia

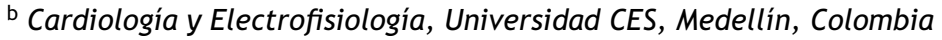

c Imagen cardiovascular, Instituto de Alta Tecnología Médica de Antioquia, Medellín, Colombia

d Residente de Radiología, Universidad CES, Medellín, Colombia

e Residente de Medicina interna, Universidad CES, Medellín, Colombia

${ }^{f}$ Epidemióloga, Universidad CES, Medellín, Colombia

Recibido el 12 de abril de 2016; aceptado el 28 de noviembre de 2016

Disponible en Internet el 9 de marzo de 2017

\section{PALABRAS CLAVE}

Fibrilación auricular; Tejido adiposo epicárdico; Grosor del tejido adiposo epicárdico; Ablación por catéter

\begin{abstract}
Resumen
Introducción: la acumulación de tejido adiposo epicárdico se asocia con obesidad y aumento en la prevalencia, severidad y recurrencia posteriores a la ablación de la fibrilación auricular. Dentro de los mecanismos implicados se considera un estado proinflamatorio que lleva al remodelamiento estructural de la aurícula y a la alteración en las propiedades electrofisiológicas de los miocitos auriculares. Hasta la fecha no se ha estudiado este fenómeno en Colombia,

Objetivo: demostrar esta asociación en un grupo de pacientes de nuestra población a quienes se les realizó ablación de venas pulmonares por radiofrecuencia.

Materiales y métodos: se revisaron 170 angiotomografías de corazón realizadas previamente a la ablación de venas pulmonares en pacientes con antecedente de fibrilación auricular. Los pacientes con datos incompletos fueron excluidos para el análisis, 94 fueron analizados. Se cuantificó el grosor del tejido adiposo epicárdico y en conjunto con el índice de masa corporal se analizó su relación con los subtipos de fibrilación auricular, así como recaídas luego de la ablación.

Resultados: la mayoría de la población se encontró con sobrepeso y obesidad. Tanto los pacientes obesos como aquellos con sobrepeso, tuvieron significativamente grosor mayor de tejido
\end{abstract}

\footnotetext{
* Autor para correspondencia.

Correo electrónico: viviq23@gmail.com (V. Quintero-Yepes).
} 
adiposo epicárdico total respecto a los pacientes con índice de masa corporal normal $(p=0,01)$. El grosor de la grasa epicárdica fue significativamente mayor en fibrilación auricular persistente respecto a la fibrilación auricular paroxística $(p=0,01)$. En los pacientes que presentaron recaídas no se observó una diferencia significativa pero sí una tendencia a tener mayor tejido adiposo epicárdico total $(p=0,08)$. El aumento del tamaño auricular se relacionó en forma significativa con el grosor del tejido adiposo epicárdico $(p=0,05)$. En el análisis de regresión logística multivariable mantuvo esta relación.

Conclusiones: el aumento del tejido adiposo epicárdico se relaciona de manera independiente con el sobrepeso y la obesidad, así como con la severidad y el pronóstico de la fibrilación auricular. Deben hacerse estudios prospectivos de cohortes en los que se evalúe y establezcan valores de corte normales para nuestra población, al igual que seguimiento a largo plazo de la evolución posterior a ablación de venas pulmonares y a intervenciones como disminución de peso.

(c) 2017 Sociedad Colombiana de Cardiología y Cirugía Cardiovascular. Publicado por Elsevier España, S.L.U. Este es un artículo Open Access bajo la licencia CC BY-NC-ND (http:// creativecommons.org/licenses/by-nc-nd/4.0/).

\section{KEYWORDS}

Atrial fibrillation; Epicardial adipose tissue;

Epicardial adipose tissue thickness;

Catheter ablation
Association of epicardial adipose tissue with atrial fibrillation and prognosis following pulmonary vein ablation

\begin{abstract}
Introduction: Accumulation of epicardial adipose tissue is associated with obesity and an increase of prevalence, severity and recurrence following ablation of atrial fibrillation. Within the implicated mechanisms, a proinflammatory state is considered when it leads to the structural remodelling of the atrium and to the alteration of the electrophysiological properties of atrial myocytes. So far this phenomenon has not been studied in Colombia.

Motivation: To prove the association in a group of patients of our population who underwent radiofrequency ablation of pulmonary veins.

Material and methods: 170 coronary CT angiographies were reviewed prior to the pulmonary vein ablation in patients with a history of atrial fibrillation. Patients with incomplete data were excluded from the analysis, 94 were analysed. Epicardial adipose tissue thickness and body mass index were measured, and their relationship with atrial fibrillation subtypes was assessed, as well as relapses following the ablation.

Results: Most participants were overweight or obese. Both obese and overweight patients had a significantly thicker total epicardial adipose tissue than those with a normal body mass index $(p=0.01)$. The thickness of epicardial fat was significantly higher in persistent atrial fibrillation in comparison with paroxysmal atrial fibrillation $(p=0.01)$. Patients with relapses did not show a significant difference but they did have a higher tendency to greater total epicardial adipose tissue. The increase in atrial size was significantly relates to the thickness of epicardial adipose tissue $(p=0.05)$. Multivariate logistic regression analysis kept this relationship.

Conclusions: The increase in epicardial adipose tissue is independently related to being overweight and obese, as well as to severity and prognosis of atrial fibrillation. Prospective cohort studies must be conducted where normal cut-off values for our population group are assessed and established, and the long-term progress following pulmonary vein ablation and other procedures such as weight loss surgery is followed.

(c) 2017 Sociedad Colombiana de Cardiología y Cirugía Cardiovascular. Published by Elsevier España, S.L.U. This is an open access article under the CC BY-NC-ND license (http:// creativecommons.org/licenses/by-nc-nd/4.0/).
\end{abstract}

\section{Introducción}

La obesidad es un problema de salud pública. Las estadísticas más recientes de la Organización Mundial de la Salud (OMS) describen que cerca del $70 \%$ de los adultos del mundo se encuentran dentro de la clasificación de sobrepeso u obesidad ${ }^{1}$. En Colombia, el $60 \%$ de la población se encuentra dentro de estos dos grupos ${ }^{2}$. La preocupación por el aumento de estas cifras se basa en sus consecuencias negativas, a sabiendas de que la obesidad es un factor de riesgo cardiovascular independiente ${ }^{3-5}$, así como también se ha demostrado su relación directa con el aumento del riesgo de desarrollar fibrilación auricular ${ }^{6-9}$. Existen mecanismos inflamatorios, autonómicos, electrofisiológicos y estructurales que sustentan la fisiopatogenia del vínculo ${ }^{10,11}$. 
Dentro de los obesos existe una población de mayor riesgo cardiovascular que son aquellos con adiposidad visceral; la acumulación de tejido adiposo epicárdico es una manifestación de ésta. Estudios clínicos han demostrado que el aumento en el tejido adiposo epicárdico se asocia con incremento de la carga aterosclerótica y del riesgo de desarrollar fibrilación auricular y de recaída posterior a la ablación por catéter $^{12-14}$. Dentro de los mecanismos implicados está el contacto directo del tejido adiposo con la superficie de la aurícula y las venas pulmonares, el cual es proveedor de mediadores inflamatorios (IL6, IL1 $\beta$ o factor de necrosis tumoral $-\alpha)^{5}$, hecho que sumado a la infiltración grasa en el epicardio y miocardio, conduce a la pérdida de la organización y homogeneidad del miocardio atrial, alterando la propagación de la onda de despolarización y favoreciendo la formación de micro-reentradas ${ }^{10}$. Adicionalmente, éste contiene abundantes plexos ganglionares, donde se integra el sistema nervioso autónomo intrínseco y extrínseco cardíaco, el cual desempeña un papel significativo en el inicio y mantenimiento de la fibrilación auricular ${ }^{13-15}$. Recientemente se demostró que en individuos con sobrepeso y fibrilación auricular sintomática, la pérdida sostenida de peso tiene un efecto dosis-dependiente en la disminución de la carga de fibrilación auricular y el mantenimiento del ritmo sinusal a largo plazo, así como en la reducción del volumen auricular izquierdo y de la hipertrofia ventricular izquierda; todo esto se explica por los cambios favorables en los factores de riesgo cardiometabólicos y disminución del estado inflamatorio ${ }^{16}$.

Se han utilizado varias técnicas no invasivas para la medición del tejido adiposo epicárdico, incluyendo ecocardiografía, imágenes por resonancia magnética o angiotomografía computada (TC); sin embargo, no existen valores de referencia, lo cual ha limitado su uso ${ }^{12,17}$.

Hasta la fecha no se había estudiado este fenómeno en Colombia. Este estudio se llevó a cabo con el fin de demostrar la asociación del aumento del tejido adiposo con la severidad de la fibrilación auricular y su recaída posterior al aislamiento eléctrico de venas pulmonares.

\section{Métodos}

\section{Población en estudio}

En este estudio retrospectivo observacional se revisaron 170 angiotomografías de corazón hechas para valorar la anatomía auricular y de las venas pulmonares en pacientes con fibrilación auricular antes de ser llevados a aislamiento eléctrico de venas pulmonares por radiofrecuencia, durante abril de 2010 a octubre de 2015.

Para el análisis de los datos se excluyeron pacientes menores de 18 años, mayores de 80 años, embarazadas y aquellos estudios con imágenes incompletas o alteraciones anatómicas que afectaron la medición del tejido adiposo epicárdico.

Se recolectaron datos de historias clínicas incluyendo edad, sexo, índice de masa corporal, historia de fibrilación auricular, subtipos de fibrilación auricular, antecedentes de tabaquismo, comorbilidades (hipertensión arterial, dislipidemia, diabetes mellitus, enfermedad coronaria), reemplazo o plastia valvular, implante de marcapasos, ablación de venas pulmonares u otras arritmias y recaída de fibrilación auricular posterior a ablación.

La definición de los subtipos de fibrilación auricular se hizo con base en las guías de fibrilación auricular de la $\mathrm{AHA} / \mathrm{ACC}^{18}$. En cuanto a clínica, se definieron como recaídas aquellos pacientes a quienes después de tres meses del procedimiento se les documentó fibrilación auricular en electrocardiograma, Holter o que tuvieron que ser sometidos a una nueva ablación.

Por otra parte, se clasificó como obesidad índice de masa corporal igual o mayor de $30 \mathrm{~kg} / \mathrm{cm}^{2}$, sobrepeso entre $25 \mathrm{y}$ $30 \mathrm{~kg} / \mathrm{cm}^{2}$ y como peso ideal menor de $25 \mathrm{~kg} / \mathrm{cm}^{2}$.

El estudio se realizó en conformidad con los principios éticos y científicos citados en la declaración de Helsinki y estuvo regido por las Buenas Prácticas Clínicas aceptadas internacionalmente y las normas vigentes de la legislación colombiana. Así mismo, fue aceptado por el comité de ética de la institución de referencia.

\section{Objetivos}

- Analizar la asociación entre el aumento del tejido adiposo epicárdico con la presencia y severidad de la fibrilación auricular.

- Dentro de los objetivos secundarios se encontraba la caracterización de la población sometida a aislamiento eléctrico de venas pulmonares, incluyendo variables sociodemográficas y clínicas.

- Considerar la relación del tejido adiposo epicárdico con la obesidad y el sobrepeso y su relación con el pronóstico posterior a la ablación de venas pulmonares como variable independiente.

\section{Protocolo de adquisición de angiotomografía}

La mayoría de los pacientes se encontraban en ritmo sinusal, excepto aquellos con diagnóstico de fibrilación auricular persistente. Utilizando un tomógrafo de 64 canales (Brilliance; Philips, Best, Holanda) se obtuvieron imágenes axiales del tórax y de alta resolución durante la fase venosa pulmonar después de la inyección de contraste yodado intravenoso. Se utilizaron $70-100 \mathrm{ml}$ de medio de contraste yodado, a una velocidad de inyección entre $4,5 \mathrm{a} 5,5 \mathrm{ml} / \mathrm{s}$, con un límite de presión de 325 psi.

Los parámetros de adquisición fueron: colimación de $64 \times 0,625$, espesor de corte $64 \times 1 \mathrm{~mm}$, tiempo de rotación del gantry 0,4 segundos, pitch 0,2 , corriente del tubo $400 \mathrm{mAs} /$ corte, potencial del tubo $120 \mathrm{Kv}$ y tiempo de scanning $9,2 \mathrm{~s}$.

Se realizó técnica de gatillado retrospectivo para sincronizar los datos con la señal electrocardiográfica (ECG). Las reconstrucciones se hicieron en fases de intervalos de $50 \mathrm{~ms}$ con un grosor de corte de $0,625 \mathrm{~mm}$ e incremento de reconstrucción de $0,5 \mathrm{~mm}$. Se escogió el intervalo de reconstrucción con la menor cantidad de artificios de movimiento y posteriormente fue usado para el análisis.

\section{Evaluación del tejido adiposo epicárdico}

Se definió tejido adiposo epicárdico (TAE) como el tejido adiposo comprendido desde la superficie miocárdica hasta 

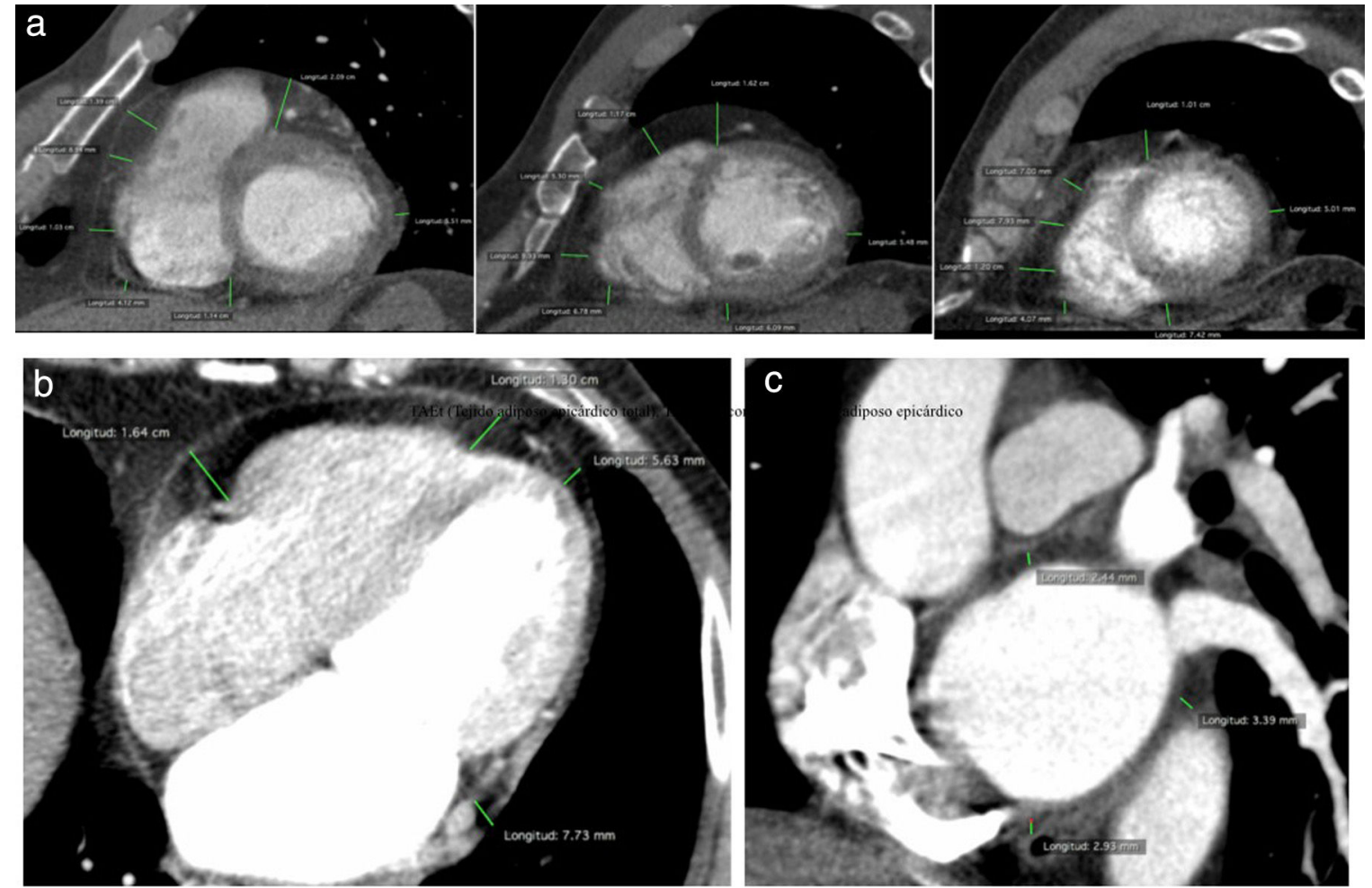

Figura 1 Cuantificación del tejido adiposo epicárdico: A) Proyección eje corto ventricular a nivel basal, medio y apical. B) Proyección 4 cámaras. C) Proyección eje corto auricular.

el pericardio parietal medido en forma perpendicular, teniendo un umbral de valores de atenuación de -30 a -250 unidades Hounsfield (UH). Las mediciones se hicieron con un cáliper digital; cada una de las imágenes fue leída por dos investigadores independientes y se calculó el promedio de estos dos valores.

Las imágenes axiales fueron trasladadas a una estación de trabajo con el software Osirix (Pixmeo SARL; Bernex, Suiza) para análisis cuantitativo. Se realizaron reconstrucciones multiplanares de los datos crudos, obteniendo las proyecciones eje largo cuatro cámaras, eje corto a nivel basal, medio y apical ventricular, así como a nivel auricular izquierdo. También se realizó cuantificación del volumen y el área auricular con el software Comprehensive cardiac analysis, de la estación de trabajo Extended Brilliance (Philips, Best, Holanda).

En la proyección eje corto se hicieron mediciones de siete segmentos a nivel basal, medio y apical: 1) Pared anterior libre superior del ventrículo derecho, 2) Pared anterior libre inferior del ventrículo derecho, 3) Pared superior del ventrículo derecho, 4) Pared diafragmática del ventrículo derecho, 5) Surco auriculoventricular superior, 6) Surco auriculoventricular inferior y 7) Pared lateral del ventrículo izquierdo (fig. 1a). En la proyección eje largo 4 cámaras se realizó medición de 4 segmentos: 1) Surco auriculoventricular derecho, 2) Surco auriculoventricular izquierdo, 3) Ápex del ventrículo derecho y 4) Ápex del ventrículo derecho (fig. 1b). La suma del valor de los segmentos medidos en la proyección eje corto a nivel basal, medio y apical con los segmentos cuantificados en la proyección eje largo 4 cámaras se definió como tejido adiposo epicárdico total
(TAEt); así mismo se definió tejido adiposo peri-coronario (TAEc) a la suma del tejido adiposo cuantificado en los surcos auriculoventricular superior e inferior en la proyección eje corto y surcos auriculoventricular derecho e izquierdo en la proyección 4 cámaras. Adicionalmente, en la proyección paraesternal corta se cuantificó el tejido adiposo periatrial, definido como la suma del tejido adiposo localizado en el espacio comprendido entre aurícula izquierda y aorta, arteria pulmonar y esófago (fig. 1c).

El área auricular se cuantificó en la proyección eje largo cuatro cámaras. Se realizó normalización por superficie de área corporal obtenida por la fórmula de Du Bois. El tamaño auricular se clasificó en normal o aumentado, según el volumen o área indexados, definiendo normal como un volumen indexado $27,3 \pm 7,9 \mathrm{ml} / \mathrm{m}^{2}$ en mujeres y $27 \pm 7,0 \mathrm{ml} / \mathrm{m}^{2}$ en hombres, y área indexada $9,3 \pm 1,7 \mathrm{~cm}^{2} / \mathrm{m}^{2}$ en mujeres $\mathrm{y}$ $8,9 \pm 1,5 \mathrm{~cm}^{2} / \mathrm{m}^{2}$ en hombres; por encima de estos valores se consideró aumentado.

\section{Análisis estadístico}

Las variables continuas se expresaron como promedio \pm desviación estándar. Las comparaciones entre los datos continuos de los pacientes se hicieron mediante la prueba $t$ de Student para muestras independientes. Los datos categóricos fueron comparados con el test de $c h i^{2}$. Se realizó una regresión logística para medir la probabilidad de recurrencia, según las variables independientes, mediante el método forward condicional; las variables con un valor de $p<0,01$ en el análisis univariado se ingresaron al modelo. Previamente 
Tabla 1 Características sociodemográficas y clínicas

\begin{tabular}{lllll}
\hline Característica & $\mathrm{n}(\%)$ & TAEt & TAE pericoronario & TAE periatrial \\
\hline Demográficas & & & & 61,2 \\
$\quad$ Género femenino & $22(23,4 \%)$ & 129,6 & 56,7 & 13,4 \\
Género masculino & $72(76,6)$ & 142,3 & & 13,3 \\
Edad (promedio en años) & 58,4 & & & 15,7 \\
Parámetros clínicos & & & 12,1 \\
Obesidad & $18(19,2)$ & 160,7 & 10,9 \\
Sobrepeso & $46(48,9)$ & 141,2 & 56,3 & 11 \\
Peso ideal & $30(31,9)$ & 123,6 & 48 & 12,9 \\
Diabetes mellitus & $10(10,6)$ & 139,4 & 58,7 & 12,4 \\
Hipertensión arterial & $49(52,1)$ & 143,2 & 58 & 13,1 \\
Enfermedad coronaria & $9(9,5)$ & 146,2 & 55,2 & 13,9 \\
Dislipidemia & $19(20,2)$ & 142,6 & 56 & 14,3 \\
Fibrilación auricular paroxística & $87(92,5)$ & 137,2 & 55,9 & 12,6 \\
Fibrilación auricular permanente & $7(7,5)$ & 165,7 & 57,4 & 14,9 \\
Recaída & $23(24,4)$ & 150,5 & 56 & 57,3 \\
Dilatación auricular & $32(34)$ & 148,2 & 56,9 & \\
Población en estudio & $94(100)$ & 139,3 & \\
\hline
\end{tabular}

TAEt: tejido adiposo epicárdico total, TAE: tejido adiposo epicárdico.

se probaron los supuestos de la regresión logística. El nivel de significancia estadística se estableció en 0,05. El análisis estadístico se efectuó con SPSS (versión 21.0, SPSS Inc., Chicago, Illinois).

\section{Resultados}

Se revisaron 170 TC hechas a pacientes con fibrilación auricular antes de ser llevados a aislamiento eléctrico de venas pulmonares. Para el análisis de variables se excluyeron 76 pacientes, los cuales tenían datos o imágenes incompletas o con alteraciones anatómicas que modificaron la medición del tejido adiposo epicárdico (37 por imágenes incompletas, 36 por datos incompletos, 1 por alteración anatómica, 2 colimación excluyó cortes necesarios para el análisis), para un total de 94 pacientes.

Las características sociodemográficas y clínicas de la población se resumen en la tabla $1(\mathrm{n}=94$, promedio de edad 58,4 años, 76,6\% fueron hombres). La mayoría de la población del estudio tuvo un índice de masa corporal mayor de $25 \mathrm{~kg} / \mathrm{m}^{2}(68,1 \%)$, con predominio de sobrepeso $(48,9 \%)$. El tejido adiposo epicárdico total fue significativamente mayor en los pacientes con obesidad y sobrepeso respecto de aquellos con peso ideal $(160,7 \pm 30,1$ y $141,2 \pm 30,3$ vs. $123,69 \pm 39,3 \mathrm{~mm}, \mathrm{p}=0,001$ ) (fig. 2). De manera similar ocurrió con los tejidos adiposos periatrial y pericoronario, los cuales fueron mayores de forma significativa en los pacientes con obesidad $(63,1 \pm 12,5$ vs. $48,06 \pm 14,2 \mathrm{~mm}, \mathrm{p}$ $=0,003$ y $15,7 \pm 7,3$ vs. $10,9 \pm 6,2 \mathrm{~mm}, p=0,029$ respectivamente) (tabla 2).

El 92,5\% de la población tenía fibrilación auricular paroxística y el 7,5\% fibrilación auricular persistente. No hubo representación de los otros tipos de fibrilación auricular en el estudio. Se observó una diferencia significativa entre el tejido adiposo epicárdico en pacientes con fibrilación auricular persistente y fibrilación auricular paroxística, siendo mayor en la primera $(165,7 \mathrm{~mm}$ vs. $137,2 \mathrm{~mm}, \mathrm{p}=0,01)$. No hubo diferencia significativa entre quienes presentaron recaídas, pero se evidenció una tendencia a mayor grosor del tejido adiposo epicárdico $(p=0,087)$. La dilatación auricular se relacionó de manera significativa con el aumento del tejido adiposo epicárdico $(p=0,053)$. No se demostró diferencia significativa entre el tejido adiposo epicárdico total y el antecedente de enfermedad coronaria, género, dislipidemia, diabetes mellitus e hipertensión arterial (tabla 3).

El análisis de regresión multivariable demostró que el tejido adiposo epicárdico se asocia de manera independiente con la recaída de la fibrilación auricular posterior al aislamiento eléctrico de venas pulmonares (IC 95\% 1,2$79, p=0,032)$; esta relación se mantuvo después de ajustar factores de riesgo de recaída como edad, hipertensión, diabetes mellitus e índice de masa corporal.

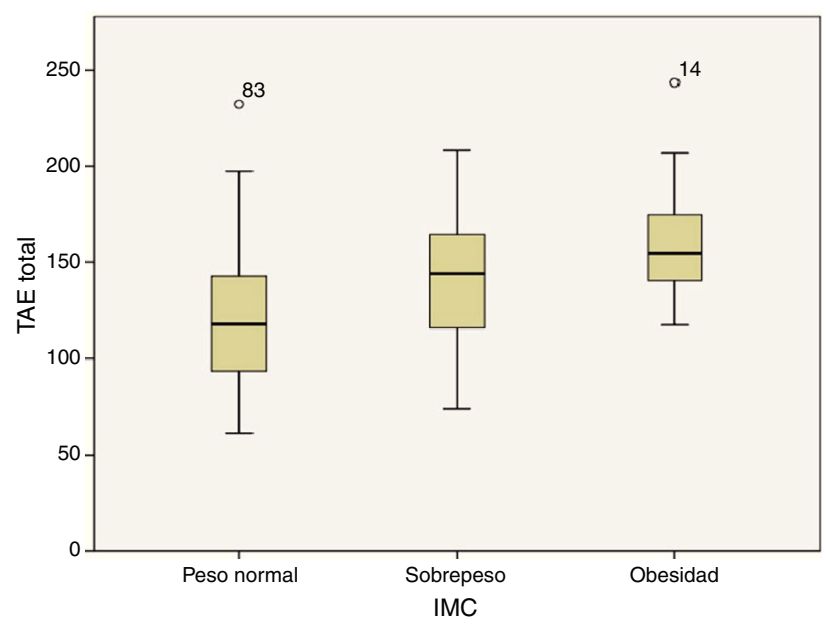

Figura 2 Relación entre índice de masa corporal y tejido adiposo epicárdico total. 
Tabla 2 Relación TAE y obesidad

\begin{tabular}{lcccc}
\hline & Obesidad $n=18$ & Sobrepeso $n=46$ & Peso ideal $n=30$ & $p$ \\
\hline TAE total (promedio) & 160,7 & 141,2 & 123,69 & 0,001 \\
TAE pericoronario & 63,1 & 56,3 & 48,06 & 0,003 \\
TAE periatrial & 15,7 & 12,1 & 10,9 & 0,029 \\
\hline
\end{tabular}

Tabla 3 Comparación entre las características basales y el tejido adiposo epicárdico total

\begin{tabular}{lll}
\hline Característica & TAEt & $\mathrm{p}$ \\
\hline $\begin{array}{l}\text { Género } \\
\quad \text { Masculino }\end{array}$ & 142,3 & 0,146 \\
$\quad$ Femenino & 129,6 & \\
Antecedente de enfermedad coronaria & & \\
$\quad \mathrm{Si}$ & 146,2 & 0,283 \\
$\quad$ No & 138,5 & \\
Antecedente de dislipidemia & & \\
$\quad$ Si & 142,6 & 0,283 \\
$\quad$ No & 138,5 & \\
Recaída de fibrilación auricular & & \\
$\quad$ Si & 150,5 & 0,087 \\
$\quad$ No & 135,3 & \\
Tipo de fibrilación auricular & & \\
$\quad$ Paroxística & 137,2 & 0,012 \\
$\quad$ Persistente & 165,7 & \\
Dilatación auricular & & \\
$\quad$ Si & & \\
No & 148,2 & 0,05 \\
\hline
\end{tabular}

TAEt: tejido adiposo epicárdico total.

\section{Discusión}

La obesidad se relaciona con un riesgo aumentado de fibrilación auricular ${ }^{9}$. En este trabajo cerca del $70 \%$ de los pacientes evaluados tenían sobrepeso y/o obesidad, con predominio del primero (48,95\%). Estudios han demostrado que el aumento del grosor del TAE se asocia con mayor prevalencia y severidad de fibrilación auricular ${ }^{19}$, así como de recaídas posteriores a aislamiento eléctrico de venas pulmonares ${ }^{5,6}$. Los hallazgos del trabajo coinciden en demostrar que el aumento en el volumen del TAE total, pericoronario y periatrial se asocia de manera significativa con el sobrepeso y la obesidad, que a su vez se relacionan con el riesgo de desarrollar fibrilación auricular. También se demostró que el TAE total fue mayor en la fibrilación auricular persistente que en la paroxística. Así mismo, el estudio mostró una tendencia a mayor cantidad de tejido adiposo epicárdico total en aquellos pacientes con recaída posterior a la ablación de venas pulmonares; sin embargo, se requiere una muestra mayor para poder demostrarlo con significancia estadística. En el análisis de regresión se mantuvo esta relación de manera independiente respecto a otros factores de riesgo de recaída.

\section{¿Es el TAE un nuevo factor de riesgo o es sólo un marcador de obesidad?}

Dada la relación directa entre obesidad y aumento del TAE, surge la inquietud acerca del papel independiente del TAE como mecanismo generador de fibrilación auricular o si éste termina siendo un marcador de la presencia de obesidad. Estudios han demostrado que más allá de los mecanismos asociados con factores de riesgo cardiovascular implicados, el aumento de la adiposidad visceral, específicamente la acumulación de tejido adiposo epicárdico, se ha asociado de forma independiente con la generación de fibrilación auricular, su severidad y pronóstico ${ }^{12,20}$, hallazgos similares a los evidenciados en nuestro estudio. Este fenómeno se ha atribuido a los efectos inflamatorios, autonómicos, electrofisiológicos y estructurales del tejido adiposo localizado en la región epicárdica ${ }^{5,13}$. En estudios previos, la acumulación del tejido adiposo en la región periatrial también se ha asociado con fibrilación auricular y riesgo de recaídas posterior a ablación ${ }^{5,20,21}$; este estudio no mostró una relación significativa, sin embargo el aumento del tamaño auricular sí se asoció con mayor grosor del tejido adiposo periatrial y su relación con la obesidad y el sobrepeso.

\section{¿Puede la disminución de peso reducir el riesgo de fibrilación auricular?}

Dada la compleja relación entre obesidad y fibrilación auricular, es válido suponer un impacto favorable en la arritmia con la reducción del peso. En un estudio con seguimiento a 5 años, se demostró cómo la disminución sostenida del peso logra una reducción en la carga de arritmia y en la recurrencia de la misma ${ }^{17}$. De manera similar, el manejo agresivo de los factores de riesgo, incluida la disminución de peso, demostró disminuir la recurrencia de arritmias en pacientes sometidos a una ablación de fibrilación auricular ${ }^{22}$. Adicionalmente, la reducción de peso también se asocia con disminución del tejido adiposo pericárdico ${ }^{23}$ situación que podría favorecer la no recurrencia de la arritmia.

\section{¿Puede la modificación del TAE impactar el riesgo de fibrilación auricular?}

Teniendo en cuenta la relación causal y consistente entre TAE y fibrilación auricular, se deben plantear alternativas para reducir el TAE y en consecuencia lograr una reducción significativa en la fibrilación auricular. La terapia intensiva con estatinas ha demostrado disminuir el TAE en pacientes con enfermedad coronaria ${ }^{24}$, así como los marcadores inflamatorios en pacientes sometidos al aislamiento eléctrico de venas pulmonares ${ }^{25}$. También se ha evaluado la 
posibilidad de realizar aislamiento eléctrico de venas pulmonares seguido de una ablación orientada a las áreas con TAE, encontrádose menor recurrencia de la arritmia en un seguimiento a 16 meses $^{26,27}$. Desde una perspectiva quirúrgica, se han estudiado las intervenciones que pretenden modificar el tejido graso epicárdico o las almohadillas grasas anteriores para lograr una modulación autonómica parasimpática (denervación cardíaca ventral-DCV-). Un metanálisis reciente concluye que la DCV en pacientes sometidos a cirugía de puentes coronarios, no reduce la incidencia de nuevos episodios de fibrilación auricular, sin embargo, otros estudios han descrito cómo la inyección de toxina botulínica en las almohadillas grasas epicárdicas en pacientes sometidos a cirugía de revascularización miocárdica, reduce la incidencia temprana y la recurrencia de fibrilación auricular hasta un año de seguimiento ${ }^{13}$. Todos estos datos sugieren un potencial impacto favorable en la prevalencia y recurrencia de la fibrilación auricular posterior al aislamiento eléctrico de venas pulmonares; no obstante, se necesitan más estudios de este tipo para que estas terapias puedan ser implementadas de forma sistemática.

\section{Limitaciones}

Por tratarse de un estudio retrospectivo se perdieron datos clínicos que no fueron consignados en los reportes médicos, lo que llevó a la exclusión de un número importante de pacientes para analizar. La población estudiada fue pequeña y sólo incluyó pacientes con fibrilación auricular paroxística y permanente. El seguimiento de las recaídas estuvo limitado por no tener un método estandarizado hecho en todos los pacientes incluidos en el estudio y por la falta de consultas de control de algunos de ellos. La medición del tejido adiposo epicárdico se realizó en dos dimensiones, así que es probable que un análisis volumétrico pudiera aportar datos más exactos y con mayor reproducibilidad; sin embargo, la técnica usada ha demostrado ser un método efectivo con bajas tasas de variabilidad intra- e interobservador ${ }^{12}$. Por último, no existen valores de corte del grosor del tejido adiposo epicárdico que permitan la clasificación de los hallazgos en patológicos o normales y por tanto esto limita su entendimiento y uso en la práctica clínica.

\section{Conclusiones}

En el ámbito local, la obesidad y el sobrepeso se relacionan con aumento del tejido adiposo epicárdico. Todos ellos se consideran predictores de severidad y recaída posterior a la ablación de venas pulmonares, por tanto la medición del índice de masa corporal y del tejido adiposo epicárdico en la angiotomografía de venas pulmonares, deberían incluirse dentro de la evaluación previa al aislamiento eléctrico de venas pulmonares.

Dada la correlación entre sobrepeso y obesidad con la acumulación de tejido adiposo epicárdico y que los cambios en el peso han demostrado mejorar estos desenlaces, se deben incluir estrategias dentro del tratamiento de la fibrilación auricular, para lograr la disminución de peso y el control agresivo de los factores de riesgo.

Deben llevarse a cabo estudios prospectivos de cohortes en los que se evalúen y establezcan valores de corte normales para nuestra población, así como seguimiento a largo plazo de la evolución posterior a ablación de venas pulmonares y a intervenciones como la disminución del peso.

\section{Responsabilidades éticas}

Protección de personas y animales. Los autores declaran que para esta investigación no se han realizado experimentos en seres humanos ni en animales.

Confidencialidad de los datos. Los autores declaran que han seguido los protocolos de su centro de trabajo sobre la publicación de datos de pacientes.

Derecho a la privacidad y consentimiento informado. Los autores han obtenido el consentimiento informado de los pacientes y/o sujetos referidos en el artículo. Este documento obra en poder del autor de correspondencia.

\section{Financiación}

Ninguna

\section{Conflicto de intereses}

Los autores declaran no tener conflictos de interés.

\section{Bibliografía}

1. WHO. Global Health Observatory (GHO): Obesity 2008. 2013.

2. WHO. Global Database on Body Mass Index.

3. Wormser D, Kaptoge S, Di Angelantonio E, Wood AM, Pennells L, Thompson A, et al. Emerging Risk Factors. Separate and combined associations of body-mass index and abdominal adiposity with cardiovascular disease: collaborative analysis of 58 prospective studies. Lancet. 2011;377:1085-95.

4. Poirier P, Giles TD, Bray GA, Hong Y, Stern JS, Pi-Sunyer FX, et al. Obesity and cardiovascular disease: pathophysiology, evaluation, and effect of weight loss. Arterioscler Thromb Vasc Biol. 2006;26:968-76.

5. Bastien M, Poirier P, Lemieux I, Despres JP. Overview of epidemiology and contribution of obesity to cardiovascular disease. Prog Cardiovasc Dis. 2014;56:369-81.

6. Goudis CA, Korantzopoulos P, Ntalas IV, Kallergis EM, Ketikoglou DG. Obesity and atrial fibrillation: A comprehensive review of the pathophysiological mechanisms and links. J Cardiol. 2015;66:361-9.

7. Magnani JW, Hylek EM, Apovian CM. Obesity begets atrial fibrillation: a contemporary summary. Circulation. 2013;128:401-5.

8. Wang TJ, Parise H, Levy D, D’Agostino RB Sr, Wolf PA, Vasan RS, et al. Obesity and the risk of new-onset atrial fibrillation. JAMA. 2004;292:2471-7.

9. Wanahita N, Messerli FH, Bangalore S, Gami AS, Somers VK, Steinberg JS. Atrial fibrillation and obesity-results of a metaanalysis. Am Heart J. 2008;155:310-5.

10. Mahajan R, Lau DH, Brooks AG, Shipp NJ, Manavis J, Wood JP, et al. Electrophysiological, electroanatomical, and structural remodeling of the atria as consequences of sustained obesity. J Am Coll Cardiol. 2015;66:1-11.

11. Abed HS, Samuel CS, Lau DH, Kelly DJ, Royce SG, Alasady $M$, et al. Obesity results in progressive atrial structural and electrical remodeling: implications for atrial fibrillation. Heart Rhythm. 2013;10:90-100. 
12. Yorgun H, Canpolat U, Aytemir K, Hazirolan T, Sahiner L, Kaya EB, et al. Association of epicardial and peri-atrial adiposity with the presence and severity of non-valvular atrial fibrillation. Int J Cardiovasc Imaging. 2015;31:649-57.

13. Liu T, Li G. Periatrial epicardial fat, local pro- and antiinflammatory balance, and atrial fibrillation. J Am Coll Cardiol. 2011;57:1249, author reply.

14. Hatem SN, Sanders P. Epicardial adipose tissue and atrial fibrillation. Cardiovasc Res. 2014;102:205-13.

15. Hatem SN. Is epicardial adipose tissue an epiphenomenon or a new player in the pathophysiology of atrial fibrillation? Arch Cardiovasc Dis. 2014;107(6-7):349-52.

16. Pathak RK, Middeldorp ME, Meredith M, Mehta AB, Mahajan R, Wong CX, et al. Long-Term Effect of Goal-Directed Weight Management in an Atrial Fibrillation Cohort: A Long-Term Follow-Up Study (LEGACY). J Am Coll Cardiol. 2015;65:2159-69.

17. Elming MB, Lonborg J, Rasmussen $T$, Kuhl JT, Engstrom $T$, Vejlstrup N, et al. Measurements of pericardial adipose tissue using contrast enhanced cardiac multidetector computed tomography-comparison with cardiac magnetic resonance imaging. Int J Cardiovasc Imaging. 2013;29:1401-7.

18. January CT, Wann LS, Alpert JS, Calkins H, Cigarroa JE, Cleveland JC Jr, et al. 2014 AHA/ACC/HRS guideline for the management of patients with atrial fibrillation: a report of the American College of Cardiology/American Heart Association Task Force on Practice Guidelines and the Heart Rhythm Society. J Am Coll Cardiol. 2014;64:e1-76.

19. Zhu W, Zhang H, Guo L, Hong K. Relationship between epicardial adipose tissue volume and atrial fibrillation: A systematic review and meta-analysis. Herz. 2015.

20. Kocyigit D, Gurses KM, Yalcin MU, Turk G, Evranos B, Yorgun $\mathrm{H}$, et al. Periatrial epicardial adipose tissue thickness is an independent predictor of atrial fibrillation recurrence after cryoballoon-based pulmonary vein isolation. J Cardiovasc Comput Tomogr. 2015;9:295-302.
21. Mahabadi AA, Lehmann N, Kalsch H, Bauer M, Dykun I, Kara $\mathrm{K}$, et al. Association of epicardial adipose tissue and left atrial size on non-contrast CT with atrial fibrillation: the Heinz Nixdorf Recall Study. Eur Heart J Cardiovasc Imaging. 2014;15: 863-9.

22. Pathak RK, Middeldorp ME, Lau DH, Mehta AB, Mahajan R, Twomey $D$, et al. Aggressive risk factor reduction study for atrial fibrillation and implications for the outcome of ablation: the ARREST-AF cohort study. J Am Coll Cardiol. 2014;64: 2222-31.

23. Abed HS, Nelson AJ, Richardson JD, Worthley SG, Vincent A, Wittert GA, et al. Impact of weight reduction on pericardial adipose tissue and cardiac structure in patients with atrial fibrillation. Am Heart J. 2015;169:655-62, e2.

24. Alexopoulos N, Melek BH, Arepalli CD, Hartlage GR, Chen Z, Kim S, et al. Effect of intensive versus moderate lipid-lowering therapy on epicardial adipose tissue in hyperlipidemic postmenopausal women: a substudy of the BELLES trial (Beyond Endorsed Lipid Lowering with EBT Scanning). J Am Coll Cardiol. 2013;61:1956-61.

25. Soucek F, Covassin N, Singh P, Ruzek L, Kara T, Suleiman M, et al. Effects of Atorvastatin $(80 \mathrm{mg})$ Therapy on Quantity of Epicardial Adipose Tissue in Patients Undergoing Pulmonary Vein Isolation for Atrial Fibrillation. Am J Cardiol. 2015;116: 1443-6.

26. Nakatani Y, Kumagai K, Minami K, Nakano M, Inoue H, Oshima S. Location of epicardial adipose tissue affects the efficacy of a combined dominant frequency and complex fractionated atrial electrogram ablation of atrial fibrillation. Heart Rhythm. 2015;12:257-65.

27. Nakahara S, Hori Y, Kobayashi S, Sakai Y, Taguchi I, Takayanagi $\mathrm{K}$, et al. Epicardial adipose tissue-based defragmentation approach to persistent atrial fibrillation: its impact on complex fractionated electrograms and ablation outcome. Heart Rhythm. 2014;11:1343-51. 